\title{
Towards Value Propositions for Persuasive Health and Wellbeing Applications
}

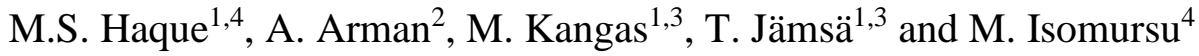 \\ ${ }^{1}$ Research Unit of Medical Imaging, Physics and Technology (MIPT), Faculty of Medicine, University of Oulu, Oulu, Finland \\ ${ }^{2}$ Department of Finance, Oulu Business School, University of Oulu, Oulu, Finland \\ ${ }^{3}$ Medical Research Center Oulu, University of Oulu and Oulu University Hospital, Oulu, Finland \\ ${ }^{4}$ INTERACT Research Group, Faculty of ITEE, University of Oulu, Oulu, Finland
}

\begin{abstract}
Recently, considerable attention has been given to health and wellbeing applications, specifically to persuasive applications. Persuasive applications refer to any interactive computing system designed to transform users' behaviours and attitudes. One of the major challenges of today's world is that health and wellbeing applications are not sustainable and scientifically designed. However, value proposition (VP) as a denominator might enhance the efficacy of the persuasive health and wellbeing applications. Research has shown little evidence on the VPs in health and wellbeing applications. This paper proposes key VPs for the persuasive health and wellbeing applications. A literature review was conducted based on relevant articles on the value within the health domain. Hence, narrative synthesis literature review approach had been used. We proposed and evaluated these VPs into our built persuasive health and wellbeing applications. We found that the VPs works well with our applications which might enhance their efficacy in the long run.
\end{abstract}

Keywords - Value co-creation, value proposition, persuasive health and wellbeing applications, case study.

\section{INTRODUCTION}

To drive health and wellbeing applications, measurement and improvement of perceived value have been suggested but the value itself remains often misunderstood [1]. If value improves, users (customers, consumers, patients, and actors etc.) can be benefitted as well as the sustainability of the applications [1]. Moreover, VPs define 'why' users will accept the application and the 'offer' [2]. To co-create value, VPs convey users and other stakeholders' solution, i.e. by involving them in one body network [3]. On Service-Dominant Logic (SDL), service or application is considered as the basis of exchange, and actors and other health service providers cocreate value. The SDL concept (application for users rather than goods) has moved into the health science literature. In recent times, persuasive applications, aimed at changing behaviours, have become well-known and popular in the health domain [4]. Persuasion is considered as an effective tool to support behaviour change [5]. Persuasive technology-oriented applications have been proposed as an actual method to encourage users' healthy lifestyle i.e. behaviour change and it has a potential for improving the quality of life [6].
Health and wellbeing applications that are available may not fulfil users' value expectations or preferences. Involving users and communities are necessary to improve health and wellbeing applications [7]. Ryhov Hospital and Jönköping County Council, Sweden initiated idea "Esther" to change health-oriented applications and represented the importance of care redesign that focuses on users' needs and preferences. They established and deployed VPs in improving their health and wellbeing applications [8]. Health application providers are facing difficulties in improving health and wellbeing applications e.g. cost and quality of applications [9]. Collaborating users and relevant stakeholders to create value is a challenge [10]. This leads to the question:

Can value propositions change persuasive health and wellbeing applications?

To answer the research question, we proposed four VPs and validated them through a case study with our built persuasive applications. Users $(\mathrm{N}=23$ for eating and $\mathrm{N}=26$ for physical activity) used the applications for one week to measure their eating and regular physical activity behavioural change. The results of this study could support to add an advantage to persuasive health and wellbeing applications, involving the users and their needs.

\section{LITERATURE REVIEW}

\section{A. Value Co-creation}

Value co-creation is the process to which health and wellbeing application providers, users, and other actors actively work together to create value for the users [11]. Co-creation of value is an interactive process to offer opportunities for the users by bringing a group of actors [12]. Value co-creation has been highlighted by SDL, which is constructed on eleven foundational premises (FP) [13]. Out of these original foundational premises, axiom FP6 (A2) and axiom FP10 (A4), was adopted in our case study since these FPs target dedicated users to determine the value (Fig. 1). Users communicate with health and wellbeing application providers at each stage of the application design and delivery [14]. 


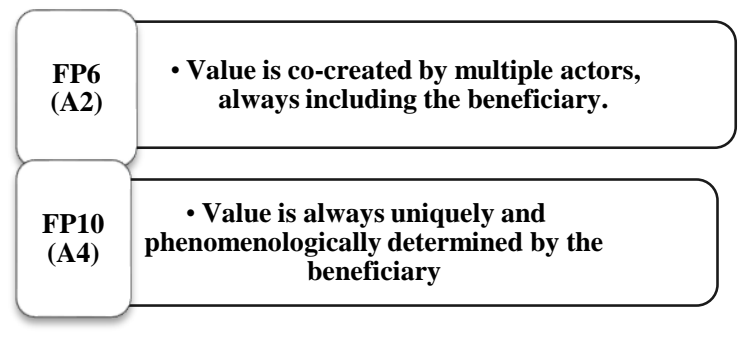

Fig. $1 S D L F P(A 2)$ and $F P 10(A 4)$

Users can sense positive experience through the value creation process of health and wellbeing applications. Co-creation of value approach meets the needs of users.

\section{B. Value Proposition (VP)}

The role of value propositions has been explored [15]. VPs can play a vital role in communicating with the users and stakeholders through initiatives and guides [16]. Health application providers offer VPs [11]. Actors cannot deliver value but participate in creation and offering of VPs [14]. This indicates that users in health and wellbeing applications participate in creating and offering VPs.

Related Work: Health Technology Assessment (HTA) [17] is widely used in the EU region to seek answers to the key issues: Does technology work? For whom does it work? What is the benefit to the individual? At what cost? How does it compare to alternatives? To address these issues, HTA consults with a range of stakeholders. HTA focuses on nine domains (health problem, technology description, clinical effectiveness, safety, cost-effectiveness, ethical concerns, organizational aspects, social impacts, legal issues) [17].

Our Approach: HTA may be a complicated approach to assess health technology. Some of the domains are closely related to our proposed VPs, e.g. technology description, cost-effectiveness and patient satisfaction. HTA does not consider particular engagement of users or scientific and empirical measurement but practiced expert validation, whereas we aimed to use theoretical concept and empirical approach.

\section{MethoD}

To identify VPs, we conducted literature search through several databases, keywords and eligibility criteria (i.e. inclusion and exclusion criteria), and verified the articles for selection for further analysis. For the inclusion criteria, we looked at the studies published in English language, accessibility with full text, and research examining the influence of values on applications focusing on health and wellbeing, and effect of values in the context of application tools to support health and wellbeing. Hence, narrative literature review approach was used and thirteen unique studies were selected for reviewing, searched for on the online repository systems: ACM Digital Library, Science Direct, Web of Science, Scopus, and EBSCO, using the combination of search terms "value", "value propositions" and "value propositions and health". The second literature search specifically targeted papers in the context of health-based information systems. From these two types of literature searches, articles were chosen based on whether they imitate value proposition in the persuasive health and wellbeing applications. The approach was conducted through careful reading of each article. Although these articles did not prompt an exact value, we matched and searched for the terms "value" and "value proposition", went through reading articles and identified whether they are useful to users. We selected the values useful to the users. The similar value was coined out from several articles and marked as a sole VP. We marked and categorized four values into four broader classes based on their relativity. Each VP was identified by resembling users' benefit within the persuasive health and wellbeing applications.

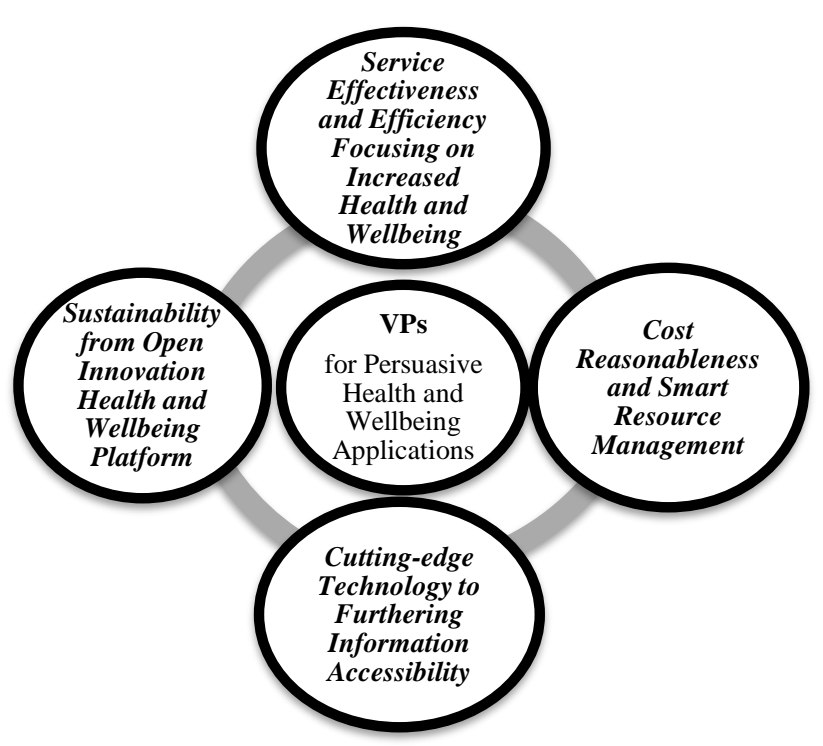

Fig. 2 VPs for persuasive health and wellbeing applications

1. Service Effectiveness and Efficiency Focusing on Increased Health and Wellbeing: An effective and efficient application includes necessary quality application provided to the users in due time [18] and communication among users and stakeholders [19][20]. Users are satisfied by perceiving higher value from the application. An effective and efficient application attracts potential users to the existing pool as well as retains existing ones. Stakeholders provide an effective 
and effective application which focuses on users' health and wellbeing and has the chance of innovation and enriching their experiences [21].

2. Cost Reasonableness and Smart Resource Management: Cost reasonableness refer to more benefits at lower cost. However, costs should not reduce to such a low level that affects the quality of the application, leading to ultimate impairment of the value of the application [22]. Costs should be reasonable per the quality of application provided [23]. Costs can be a combination of monetary costs, psychic costs and time costs [24]. Monetary cost is the money spent for the generation of the application. Lower monetary cost helps the user to choose economically beneficial health and wellbeing applications. Psychic cost is related to mental dissatisfaction resulting from extensive and tiresome application procedure [24]. Smart resource management means a scientifically effective and efficient use of available resources to create highquality applications [18].

3. Cutting-edge Technology to Furthering Information Accessibility: Users require proper access to necessary information. Not all relevant stakeholders should have access to all information. Rather specific information should be made available to a specific type of stakeholders, e.g. users, health care professionals and specialists need information access [25][26] and professionals need timely access to personal health records of their patients/users [20][27]. Using cuttingedge technology, users and relevant stakeholders get timely access to information with confirmed security and privacy.

4. Sustainability from Open Innovation Health and Wellbeing Platform: Shifting from traditionally closed innovation to open innovation, i.e. to open source approach, has been recommended for user-oriented health and wellbeing applications [27]. Open innovation health and wellbeing platform may bring interesting outcomes, e.g. novel concepts and solutions in health and wellbeing applications that are dedicated for the users. Scholars also emphasized on the empathic support and exchange of information as important elements of communication [28]. Open innovation helps the application providers to reduce costs [29] that might encourage users to use the application. Moreover, it helps users to get the most recently innovated application in an easy and convenient way because application providers constantly change and upgrade their applications based on the needs of users [30].

\section{CAse study}

To evaluate our proposed VPs, we analyzed two developed persuasive health and wellbeing applications, iCrave to promote healthy eating [31], and iGO to promote physical activity [32]. Both applications are designed with an aim that users are satisfied by perceiving higher value from the application, i.e. value in a sense of their healthy eating and physical activity (PA) promotion. The applications we designed so that the users can have a positive experience in terms of their eating and PA. This relates to the fulfilment of the first VP. These applications are free to use for the users, i.e. the users can download them from the Android store for a smartphone or they can use the applications on a website. The applications are designed as user-friendly and simple to operate, i.e. maintaining the psychic cost. This is related to fulfilment of the second VP. We applied gamified and persuasion technique within the applications, adding some game elements such as points, badges etc. For the healthy eating application iCrave, we applied a $\mathrm{QR}$ code to be scanned by the phone. For the PA promotion app iGO, we applied an algorithm that allows the sensor of the smartphone to track walking steps. Users can insert their information when logging in, and they can monitor their daily activities. The admin can only access users' data once received consents from them. This reflects the fulfilment of the third VP. Lastly, to design the applications we searched the literature for the established theories which can be applied to the application and tested empirically. The healthy eating app iCrave was designed by incorporating the EI intrusion theory and iGO was designed by incorporating the SDT theory, and the design followed the user-centered design (UCD) process. The applications were tested empirically by end users to gather information in relation to the apps upgrade, usability issues etc. Users found the applications to motivate themselves in healthy eating and PA, which implies the fourth VP.

A method is recommended to involve users and relevant stakeholders for a value co-creation model [33]. Our approach in listing the VPs for persuasive health and wellbeing applications might be one successful method since the users and other actors are involved. Our approach to finding the VPs of the health and wellbeing applications is a combination of collecting information on the individual user and other relevant stakeholders' experience, quality and cost of health and wellbeing applications to increase the applications' efficacy and involving users to co-create the value. However, our proposed VPs are limited to literature and expert validation, such as HTA core model does, is not involved. Further study needs more concentration on the extract version of these four VPs to highlight their importance for different types of users. In future, expert validation of VPs should be performed.

\section{CONCLUSIONS}

We integrated the theoretical concept of value co-creation to gauge the VPs. The VPs derived from the literature are described. We combined VPs derived from literature and integrated them by finalizing four key VPs. We performed a 
case study to evaluate the proposed VPs, showing VPs being a successful approach. Health application providers and other relevant stakeholders should work together to provide high quality and efficient health and wellbeing applications to users.

\section{CONFLiCT OF INTEREST}

The authors declare that they have no conflict of interest.

\section{REFERENCES}

1. Porter, M.E. (2010). What is Value in Healthcare? The New England Journal of Medicine, 363, pp.2477-2481

2. Lindic, J. and Silva, C.M. (2011). Value proposition as a catalyst for a customer focused innovation. Management Decision, 49, pp.1694-1708

3. Gummesson, E. (2008). Customer centricity: reality or a wild goose chase? European Business Review, 20(4), pp.315-330

4. Chatterjee, S. and Price, A. (2009). Healthy Living with Persuasive Technologies: Framework, Issues, and Challenges. Journal of the American Medical Informatics Association, pp.171-178

5. O'Keefe, D.J. (2002). Persuasion theory and research (2nd. Sage Publications)

6. Fogg, B.J. (2003). Persuasive Technology: Using Computers to Change What We Think and Do. San Fransisco: Morgan Kaufm

7. KPMG. (2014). Creating new value with parents, [Online] Available:<www.kpmg.com/Global/en/IssuesAndInsights/Artic lesPublications/what-works/creating-new-value-with-patients [11.04.2016]

8. Schnarr, S. and Alessi. C. and Schnarr. K. (2014). It's all about me: The Personalization of Health Systems. Ivey Business School

9. Vargo, S.L., Maglio, P. and Akaka, M. (2008). On value and value co-creation: A service systems and service logic perspective. European Management Journal, 26(3), pp.145-152

10. Grönroos, C. (2008). Service logic revisited: who Creates Value? And who co-creates? European Business Review, 20(4)

11. Gronroos, C. and Vioma, P. (2013). Critical service logic: making sense of value creation and co-creation. Acad. Mark. Sci. $41, \mathrm{pp} .133-150$

12. Jacob, C. (1992). A power primer. Psychological Bulletin, 112(1)

13. Vargo, S.L. and Lusch, R.F. (2016). Institutions and axioms: an extension and update of service-dominant logic. Journal of the Academy of Marketing Science

14. Ballantyne, D. (2004). Dialogue and its role in the development of relationship-specific knowledge. Journal of Business and Industrial Marketing, 19(2), pp.114-123

15. Frow, P. and Payne, A. (2011). A Stakeholder Perspective of the Value Proposition Concept. European Journal of Marketing, 45, pp.233-240

16. Ballantyne, D., Frow, P., Varey, R. and Payne, A. (2011). Value Propositions as Communication Practice: Taking a Wider View. Industrial Marketing Management, 40(2), pp.202-210

17. HTA Core-Model. (2014). [Online] Available from :<www.hiqa.ie/HTA-GuidelinesStakeholderManagement [11.07.2016]
18. Huerta, T.R., Ford, E.W, Peterson, L.T. and Brigham, K.H (2008). Testing the hospital value proposition: An empirical analysis of efficiency and quality. Health Care Manage Rev, 33(4), pp.341-349

19. Pilon, B., Crutcher, Terri, D., Lemmin-Lee, S., Watters, R., Wolgast, K.A. and Arnow, D. (2014). The value proposition for Graduate education of emerging nurse leaders: Immediate Benefit to Organizations. Nurse Leader, 12(3), pp.81-85

20. Kaelber, D.C., Jha, A.K., Johnston, D., Middleton, B. and Bates, D.W. (2008). A research agenda for personal health records (PHRs). Journal of the American Medical Informatics, 15(16)

21. Abidi, S.S.R. (2007). Healthcare knowledge sharing: purpose, practices, and prospects. Healthcare Knowledge Management, pp.67-86. Springer

22. Brown, S. and Saint, M. (2013). Value Proposition for mHealth Monitoring Solution of Diabetes. IST-Africa 2013 Proceedings, IIMC International Information Management Corporation

23. Krey, M., Bettina, H., Matthias, K. and Steven, F. (2010). IT Governance, Risk Management and Compliance in Swiss Healthcare. Proceedings of the UKSim 12th International Conference on Computer Modelling and Simulation, pp.340-345

24. Narayan, G. and Nerurkar, A. (2006). Value-proposition of egovernance services: Bridging rural-urban digital divide in developing countries. International journal of education and development using ICT

25. Helman, D., Addeo, E. and Walters, D. (2011). Ubiquity and integration in m-Health: implications for brand management. In: Sarnoff Symposium

26. Manary, M., Boulding, W., Staelin, R.W. and Glickman, SW. (2013). The patient experience and health outcomes. N Engl J Med, 368, pp.201-203

27. Lundberg, N., Koch, S., Hägglund, M., Bolin, P., Davoody, N., Eltes, J., Jarlman, Perlich, A., O., Vimarlund, V. and Winsnes, C. (2013). Mycare pathways: creating open innovation in healthcare. MEDINFO, pp.687-691

28. Bullinger, A.C., Rass, M., Adamczyk, S., MOeslein, K.M. and Sohn, S. (2012). Open innovation in health care: Analysis of an open health platform. Health Policy, 105(2-3), pp.165-175

29. Huizingh, E.K. (2011). Open innovation: State of the art and future perspectives. Technovation, 31(1), pp.2-9

30. Reinhardt, R., Bullinger, A.C. and Gurtner, S. (2015). Open innovation in health care. Challenges and Opportunities in Health Care Management, pp.237-246

31. Hsu, A., Yang, J., Yilmaz, Y., Haque, Md., Cengiz, C. and Blandford, A. (2014). Persuasive technology for overcoming food cravings and improving snack choices. Proceedings of ACM CHI, 2014, Ontario, Canada

32. Haque, Md., Abdullah, W., Rahman, S., Kangas, M. and Jämsä, T. (2016). Persuasive health and wellbeing application: A theorydriven design in promoting physical activity. Proceedings of IEEE MediTec, 2016, Dhaka, Bangladesh

33. Demiris, G. and Kneale, L. (2015). Informatics systems and tools to facilitate patient-centered care-coordination. Yearbook of medical informatics, 10(1)

Author: Md. Sanaul Haque

Institute: Research Unit of MIPT, University of Oulu

Street: P.O. Box. 5000, 90014 Oulu

City: Oulu

Country: Finland

Email:md.haque@oulu.fi 